\title{
Radioisotope measurements of the liquid-gas flow in the horizontal pipeline using phase method
}

\author{
Robert Hanus ${ }^{1, *}$, Marcin Zych ${ }^{2}$, Marek Jaszczur $^{3}$, Leszek Petryka ${ }^{4}$, and Dariusz Świsulski ${ }^{5}$ \\ ${ }^{1}$ Rzeszów University of Technology, Faculty of Electrical and Computer Engineering, 35-959 Rzeszów, Poland \\ ${ }^{2}$ AGH University of Science and Technology, Faculty of Geology, Geophysics and Environmental Protection, 30-059 Kraków, Poland \\ ${ }^{3}$ AGH University Science and Technology, Faculty of Energy and Fuels, 30-059 Kraków, Poland \\ ${ }^{4}$ AGH University of Science and Technology, Faculty of Physics and Applied Computer Science, 30-059 Kraków, Poland \\ ${ }^{5}$ Gdańsk University of Technology, Faculty of Electrical and Control Engineering, 80-233 Gdańsk, Poland
}

\begin{abstract}
The paper presents application of the gamma-absorption method to a two-phase liquid-gas flow investigation in a horizontal pipeline. The water-air mixture was examined by a set of two Am-241 radioactive sources and two $\mathrm{NaI}(\mathrm{Tl})$ scintillation probes. For analysis of the electrical signals obtained from detectors the cross-spectral density function (CSDF) was applied. Results of the gas phase average velocity measurements for CSDF were compared with results obtained by application of the classical crosscorrelation function (CCF). It was found that the combined uncertainties of the gas-phase velocity in the presented experiments did not exceed $1.6 \%$ for CSDF method and $5.5 \%$ for CCF.
\end{abstract}

\section{Introduction}

Two-phase liquid-gas flows - when gas is transported by liquid - commonly occurs in industry, especially in mining, nuclear, pharmaceutical, petrochemical, energy as well as in environmental engineering. This type of flows are of particular importance in describing heat transfer and mass processes in a number of devices such as, reactors, distillation columns, heat exchangers, absorption and rectification columns.

Two-phase flows are very difficult for detailed the mathematical description. Therefore, experimental research and the development of the new methods for measuring such flows are of major importance. Twophase flows can be studied using several methods, such as computer tomography, particle image velocimetry (PIV), magnetic resonance imaging (MRI), laser Doppler anemometry (LDA) or using Coriolis flowmeters, optical and nuclear techniques [1-11]. The nuclear methods use radiotracers or sealed gamma-ray sources and scintillation detectors for the flow analysis. The gammaabsorption method is particularly convenient for continuous investigation of two-phase flows. The absorption measurement is relatively safe, non-invasive, and allows determining the velocity as well as concentration of a dispersed phase using the same set-up. Most important in such measurements is the analysis of the electrical signals received from the probes. Due to the stochastic nature of obtained signals, statistical methods have to be used for this purpose. The measured time delay of signals is applied to determine the averaged velocity of the dispersed phase and other flow parameters.
The most known methods of time delay estimation for stationary random signals is the cross-correlation function (CCF) $[8,12]$. Other methods (in time domain) include differential and combined methods [13, 14], Hilbert Transform-based CCF $[15,16]$ or methods based on the conditional averaging of signals $[17,18]$. In the frequency domain the phase of cross-spectral density function (CSDF) can be used [19,20]. This paper presents an application of the phase method to evaluate the mean velocity of gas phase in the two phase gaswater mixture in a horizontal pipe.

The paper consists of five sections. In Section 2 the basis of gamma-absorption method for liquid-gas flow evaluation and the laboratory set-up are described. Section 3 presents principles of time delay estimation applying phase of CSDF. In section 4 the comparison between results of average velocity measurements obtained using phase method and CCF are presented. The last section contains the final conclusions.

\section{Gamma-absorption method and laboratory set-up}

The gamma absorption technique is based on exponential decay of a gamma radiation beam in function of composition and geometry of the absorbent [9]. The changes of the intensity of radiation are detected by the scintillation probes and converted into electrical impulses [7].

The typical gamma-absorption set for two-phase liquid-gas flow measurement is presented in Fig. 1. Two sealed radioactive sources emit gamma radiation beams shaped by collimators (1). Photons pass through the pipe

\footnotetext{
Corresponding author: rohan@prz.edu.pl
} 
and two-phase flows mixture (4). Two scintillation detectors (2) with collimators (3) are placed on the other in referent to the suorces side of the pipe at $L=97 \mathrm{~mm}$ distance between them. In the presented experiment two sets consisting of linear Am-241 sources with an activity of $100 \mathrm{mCi}$ and probes with $\mathrm{NaI}(\mathrm{Tl})$ scintillation detectors were used. At the outputs of two scintillation probes, count signals $I_{x}(t)$ and $I_{y}(t)$ are registered for the first and second probe respectively [21].

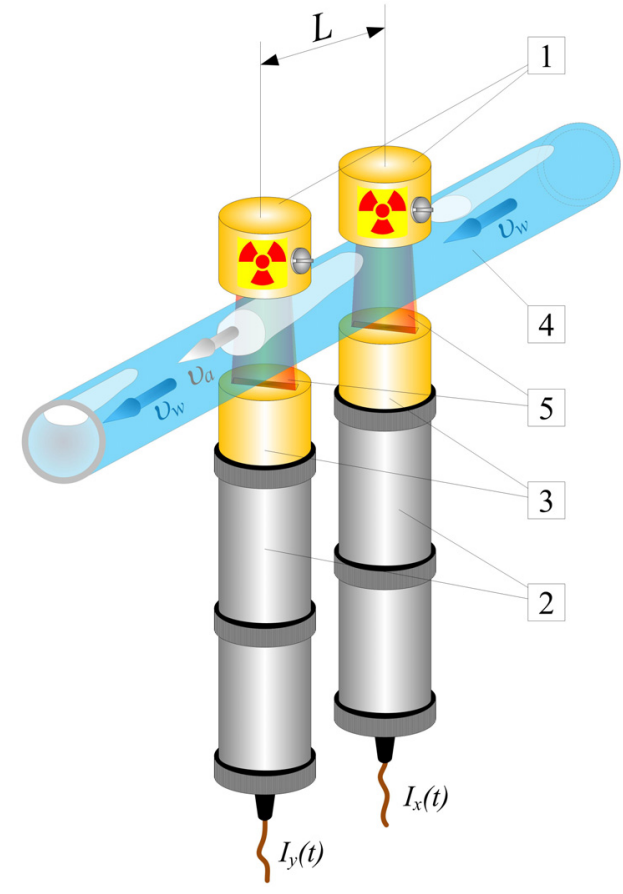

Fig. 1. The gamma-absorption measuring set: 1 - gamma ray sources with collimators, 2 - scintillation probes, 3 - detectors' collimators, 4 - pipeline, 5 - the main beam of gamma rays, $v_{a}-$ velocity of air, $v_{W}-$ velocity of water. [21]

The gamma-absorption equipment described above was applied in the experimental measurement set-up, performed in Industrial Radiometry Laboratory of the AGH University of Science and Technology in Krakow, Poland. Measurement data waere collected during experiments carried out on a laboratory hydraulic installation with a horizontal plexiglas pipe of $4.5 \mathrm{~m}$ length and inner diameter of $30 \mathrm{~mm}$. A detailed description of experimental set-up can be found in [16, 18 ]. Figure 2 shows the general view of the measurement section.

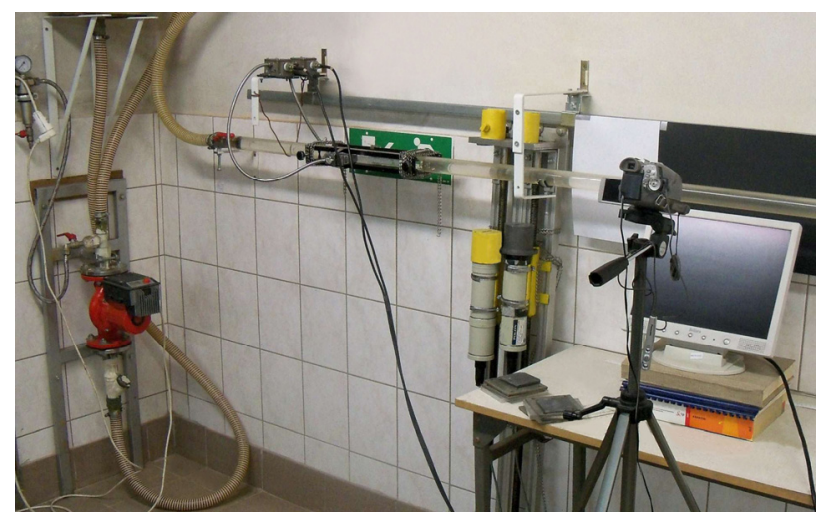

Fig. 2. General view of the experimental set-up.
The data acquisition system include a dedicated 8channel counter - HSC 8000 (EC Electronics) connected to work station using an USB port. Voltage pulses $I_{x}$ and $I_{y}$ are counted within the sampling time $\Delta t=1 \mathrm{~ms}$ and create mutually delayed discrete stochastic signals $x$ and $y$. An exemplary time records of signals obtained in the LIT0003 experiments are presented in Fig. 3.
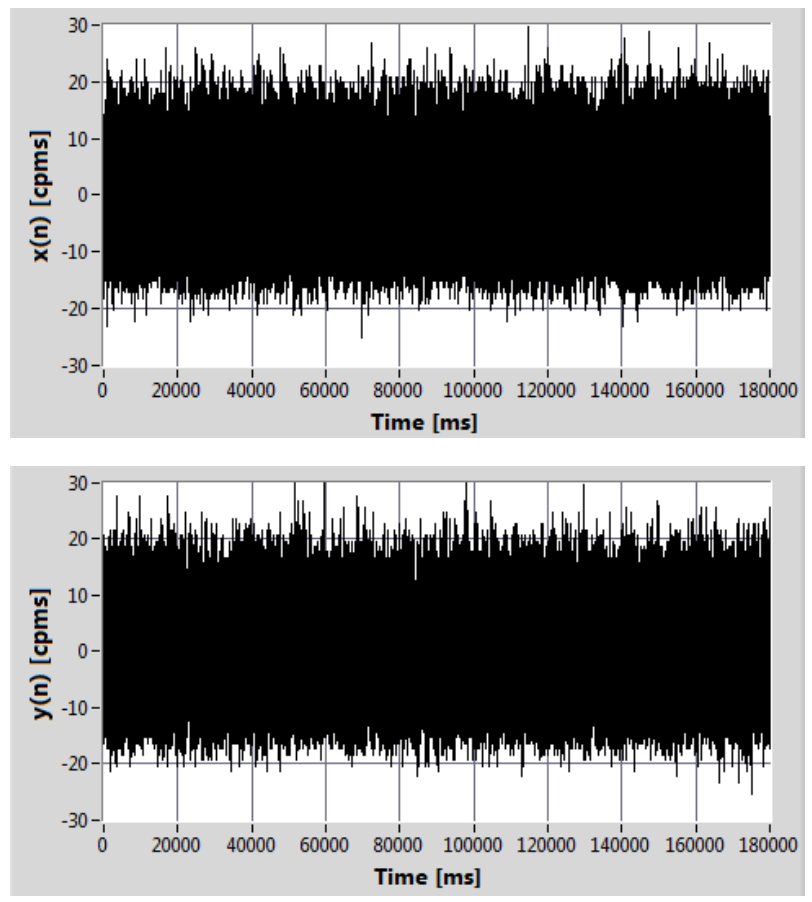

Fig. 3. Time records of signal $x(t)$ and $y(t)$ (after centering) obtained in the LIT0003 experiment.

\section{Analysis of signals}

The analyses of signals from scintillation probes in the time or frequency domain allow determining of the transportation time delay $\tau_{0}$ and then the mean velocity $v_{a}$ of gas phase transportation:

$$
v_{a}=L / \tau_{0}
$$

The most known classical method of time delay estimation of random signals is based on the CCF and defined as follow [12]:

$$
R_{x y}(\tau)=\lim _{T \rightarrow \infty} \frac{1}{T} \int_{0}^{T} x(t) y(t+\tau) d t
$$

where $T$ is the averaging time and $\tau$ is the time delay.

The $\tau_{0}$ transportation time delay is determined by finding the location for the CCF maximum.

Time delay estimation in the frequency domain is possible by use of the phase of CSDF. In practice only one-sided CSDF for the frequency range $0<f<\infty$ can be designated. This is a complex quantity defined as follows [19]:

$$
\underline{G}_{x y}(f)=2 \int_{-\infty}^{\infty} R_{x y}(\tau) \cdot e^{-j 2 \pi f \tau} d \tau
$$


Normalized CSDF is referred to as coherence function $\gamma_{x y}(f)$. In practice, the real-valued squared coherence function is used:

$$
\gamma_{x y}^{2}(f)=\frac{\left|\underline{G}_{x y}(f)\right|^{2}}{G_{x x}(f) \cdot G_{y y}(f)}
$$

where $G_{x x}(f)$ and $G_{y y}(f)$ are autospectral density functions of signals $x(t)$ and $y(t)$ respectively.

The squared coherence $\gamma_{x y}^{2}(f)$ satisfies the condition: $0 \leq \gamma_{x y}^{2}(f) \leq 1$.

The $\Phi_{x y}(f)$ phase of the CSDF is a linear function of frequency $f$ :

$$
\Phi_{x y}(f)=\operatorname{arctg}\left\{\operatorname{Im}\left[G_{x y}(f)\right] / \operatorname{Re}\left[G_{x y}(f)\right]\right\}=2 \pi \cdot f \cdot \tau_{0}(5)
$$

Currently most often the discrete Fourier transform (DFT) is applied to calculate the spectral densities directly for the discrete signals $x(n)$ and $y(n)$, where $n=t / \Delta t$. In analysis of stochastic signals an averaging of the obtained spectral density estimators is necessary. The most popular Welch procedure requires the sets of signal samples to be divided into $N_{d}$ windowing segments, each of the $N$ samples. The $N_{d}$ and $N$ values should be big enough to limit the statistical fluctuations of CSDF estimation [19]. DFT gives the $k$ useful frequencies $f_{k}(k=0 \ldots(N-1) / 2)$, with the spectral resolution $\Delta f=1 / N \Delta t$. Consequently the transportation time delay $\tau_{0}$ is determined from the smoothed $\widetilde{\Phi}_{x y}(f)$ estimator after applying the procedure of the phase unwrapping [22]. Figure 4 presents the plot of the $\widetilde{\Phi}_{x y}(f)=f(f)$ function determined in the LIT0003 experiment $\left(N_{d}=533, N=500\right.$, overlapping $35 \%$ and Hanning window).

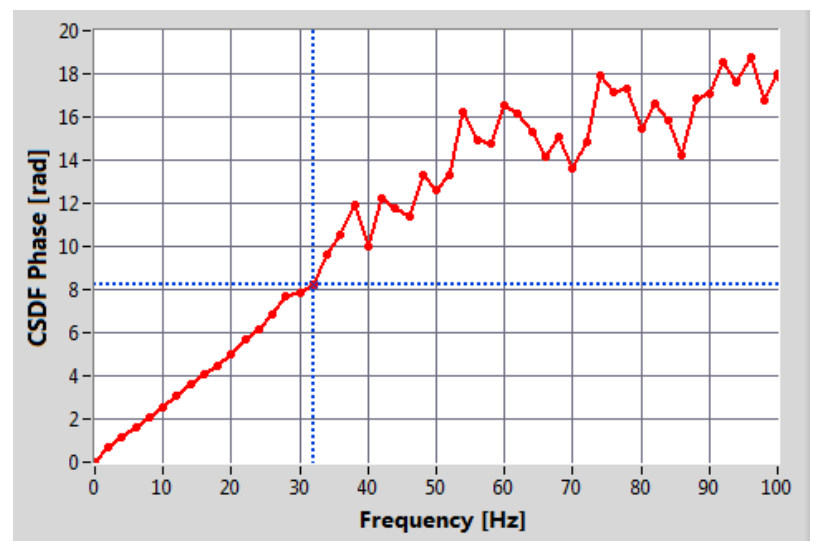

Fig. 4. Plot of the smoothed CSDF phase.

The function $\widetilde{\Phi}_{x y}(f)$ is nominally linear and passes through the coordinate origin, so the $\hat{\tau}_{0}$ time delay estimator can be determined by use slope $a$ of the regression line fitted to the CSDF phase:

$$
\hat{\tau}_{0}=\frac{1}{2 \pi} \cdot \hat{a}=\frac{1}{2 \pi}\left[\sum_{k=1}^{m} f_{k} \cdot \widetilde{\Phi}_{x y}\left(f_{k}\right) / \sum_{k=1}^{m} f_{k}^{2}\right]
$$

where $m$ is the number of the pairs of both phase and frequency values used for the regression analysis [20, 23].

The selection of the appropriate frequency linearization range is vital due to fluctuations of the CSDF phase above the cut-off frequency band of the recorded signals. In this case the cut-off frequency can be determined based on the significant value of coherence function [20]. Figure 5 presents the graph of the smoothed coherence $\widetilde{\gamma}_{x y}^{2}(f)$ obtained in the LIT0003 experiment.

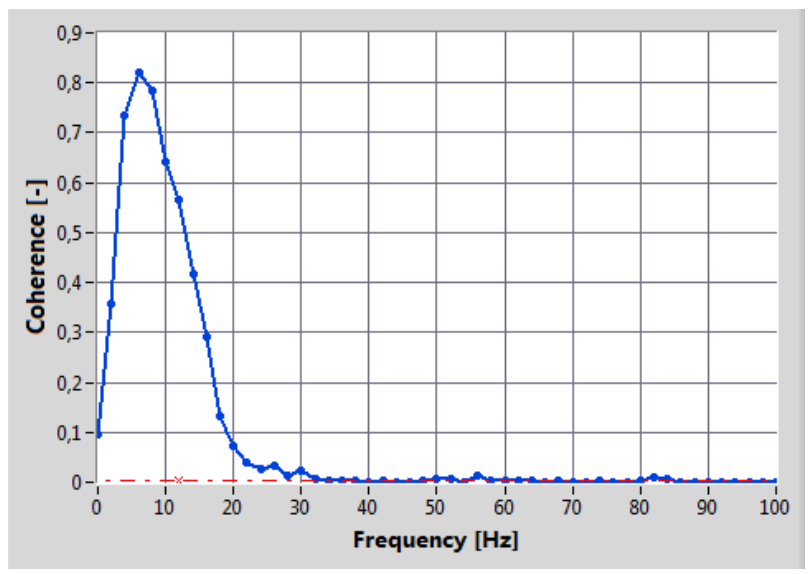

Fig. 5. Smoothed coherence function.

The dotted red line in the Fig. 5 marks the border of onesided confidence interval $E_{\alpha}$ fixed for the significance level $\alpha=0.05$ from the relation [24]:

$$
E_{\alpha}=(1-\alpha)^{1 /\left(N_{d}-1\right)}
$$

Based on the $E_{\alpha}$ value, the cut-off frequency and $m$ value for $\hat{\tau}_{0}$ time delay estimation are determined.

\section{Exemplary results}

The plot of the CSDF phase obtained in the LIT0003 experiment for the selected frequency range from 0 to $32 \mathrm{~Hz}$ is shown in Fig. 6. The solid line represents the fitted regression line determined to find out the transportation time delay estimator from eq. (6).

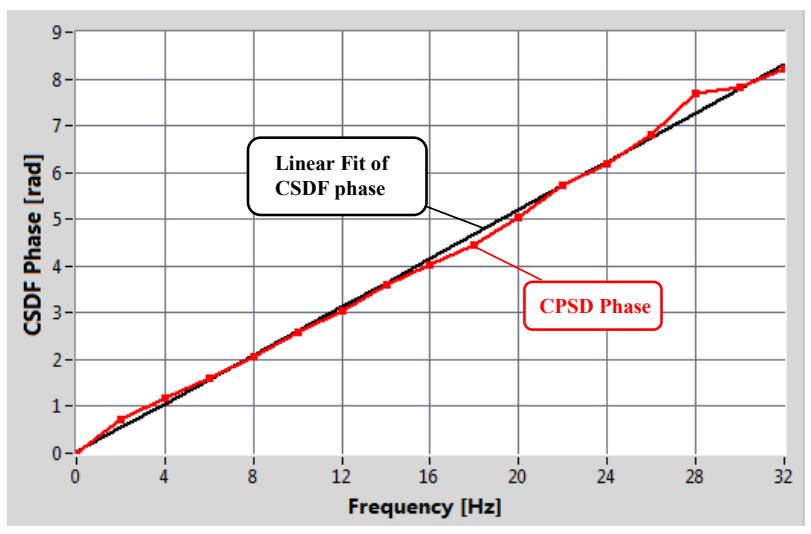

Fig. 6. A linear fit of the smoothed CSDF phase determined in the LIT0003 experiment. 
The standard uncertainty $u\left(\hat{\tau}_{0}\right)$ calculated using CSDF phase is given by $[20,23]$ :

$$
u\left(\hat{\tau}_{0}\right)=\left\{\frac{1}{4 \pi^{2}(m-1) \sum_{k=1}^{m} f_{k}^{2}}\left[\sum_{k=1}^{m} \widetilde{\Phi}_{x y k}^{2}\left(f_{k}\right)-\frac{\left[\sum_{k=1}^{m} f_{k} \cdot \widetilde{\Phi}_{x y k}\left(f_{k}\right)\right]^{2}}{\sum_{k=1}^{m} f_{k}^{2}}\right]\right\}^{1 / 2}
$$

Assuming negligible small uncertainties of the data acquisition system, the combined standard uncertainty $u_{c}\left(v_{a}\right)$ of the gas phase average velocity depends on an inaccuracy of uncorrelated $L$ and $\hat{\tau}_{0}$ determination [25]:

$$
u_{c}\left(v_{a}\right)=\left[\left(\frac{\partial v_{a}}{\partial L}\right)^{2} \cdot u^{2}(L)+\left(\frac{\partial v_{a}}{\partial \hat{\tau}_{0}}\right)^{2} \cdot u^{2}\left(\hat{\tau}_{0}\right)\right]^{1 / 2}
$$

where $u(L)$ is the standard uncertainty of the distance between detectors.

In table 1 the results of the average velocity $v_{a}$ and its combined uncertainties $u_{c}\left(v_{a}\right)$ obtained in the selected LIT experiments by the use of CSDF phase method are presented. For comparison, the results obtained under analysis of the CCF distribution are also given.

Table 1. Exemplary results of experiments.

\begin{tabular}{|c|c|c|c|}
\hline Method & $\begin{array}{c}\text { Average } \\
\text { velocity } \\
v_{a}[\mathrm{~m} / \mathrm{s}]\end{array}$ & $\begin{array}{c}\text { Combined } \\
\text { uncertainty } \\
u_{c}\left(v_{a}\right)[\mathrm{m} / \mathrm{s}]\end{array}$ & Experiment \\
\cline { 1 - 3 } $\begin{array}{c}\text { CSDF } \\
\text { phase }\end{array}$ & 1,781 & 0,016 & \multirow{2}{*}{ LIT0002 } \\
\cline { 1 - 3 } CCF & 1,798 & 0,093 & \\
\hline $\begin{array}{c}\text { CSDF } \\
\text { phase }\end{array}$ & 2,314 & 0,015 & \multirow{2}{*}{ LIT0003 } \\
\cline { 1 - 3 } CCF & 2,295 & 0,125 & \\
\hline $\begin{array}{c}\text { CSDF } \\
\text { phase }\end{array}$ & 2,679 & 0,044 & \multirow{2}{*}{ LIT0004 } \\
\cline { 1 - 2 } CCF & 2,712 & 0,144 & \\
\hline
\end{tabular}

\section{Conclusions}

This paper presents an application of the CSDF phase method to radioisotope measurement of the velocity of gas phase in water-air mixture transportation by horizontal pipeline. It was found that the combined uncertainties of the average velocity of air in the exemplary LIT experiments did not exceed $1.6 \%$ for the CSDF phase method and $5.5 \%$ for the CCF method respectively. Moreover, in the cross-correlation method, the filtration of the recorded signals should be applied in order to reduce noises [16]. On contrary to CCF method, the filtration of signals in the phase method is not necessary.

Based on the obtained results, it can be stated that the CSDF phase method may be successfully applied in investigation of liquid-gas flow in horizontal pipeline in connection with gamma-ray absorption method.

\section{References}

1. M. Bottin, et al, Int. J. Multiphas. Flow 60, 161-179 (2014)

2. T. Xue, et al, Flow Meas. Instrum. 27, 29-36 (2012)

3. G. Falcone, G.F. Hewitt, C. Alimonti, Multiphase flow metering: principles and applications (Elsevier, Amsterdam 2009)

4. R.L. Powell, Phys. Fluids. 20, 040605 (2008)

5. I. Ismail, et al, Flow Meas. Instrum. 16, 145-155 (2005)

6. G.A. Johansen, P. Jackson, Radioisotope gauges for industrial process measurements (John Wiley, New York 2004)

7. Z. Yu, B. Qincheng, H. Richa, Appl. Therm. Eng. 60, 398-410 (2013)

8. S.L. Soo (ed.), Instrumentation for fluid-particle flow (Noyes Publications, New Jersey, 1999)

9. W.A.S. Kumara, B.M. Halvorsen, M.C. Melaaen, Int. J. Multiphas. Flow 36, 467-480 (2010)

10. V. Mosorov, Meas. Sci. Technol. 17, 753-760 (2006)

11. P. Vlasak, et al, J. Hydrol. Hydrom. 62, 241-247 (2014)

12. M.S. Beck, A. Plaskowski, Cross correlation flowmeters - their design and application. (Adam Hilger, Bristol, 1987)

13. G. Jacovitti, G. Scarano, IEEE Trans. Sign. Proc 41, 525-533 (1993)

14. R. Hanus, et al., Math. Probl. Eng. 2014, 475735 (2014)

15. J.S. Bendat, The Hilbert Transform and applications to correlation measurements Brüel\&Kjær BT0008-11 (Naerum, Denmark, 1985)

16. R. Hanus, Int. J. Multiphas. Flow 72, 210-217 (2015)

17. A. Kowalczyk, Measurement applications of conditional signal averaging (O.W. Politechniki Rzeszowskiej, Rzeszów, 2015) (in Polish)

18. R. Hanus, et al, EPJ WoC 92, 02021 (2015)

19. J.S. Bendat, A.G. Piersol, Random data - analysis and measurement procedures (John Wiley, New York, 2010)

20. R. Hanus, et al, EPJ WoC 92, 02020 (2015)

21. R. Hanus, et al, EPJ WoC 143, 02033 (2017)

22. R.G. Lyons, Understanding digital signal processing (Prentice Hall, New York, 2011)

23. A.G. Piersol, IEEE Trans. Acoust. Speech Sign Proc. 29, 471-477 (1981)

24. D.M. Halliday, et al, Prog. Biophys. Mol. Bio. 64, 237-278 (1995)

25. Guide to the expression of uncertainly in measurement (International Organisation for Standardisation, 1995) 